\title{
Experimental analysis of storage and retrieval processes involved in retroactive inhibition: The effect of presentation mode
}

\author{
Karl-Heinz Bäuml * \\ Regensburg University, Regensburg, Germany
}

Accepted March 1991

\begin{abstract}
While there is strong empirical evidence in the literature that, without interpolation, massed or distributed presentation of categorizable word pairs affects both storage and retrieval processes, theories of retroactive inhibition tacitly assume that presentation mode does not play a substantial role with interpolation. By means of Batchelder and Riefer's (1980) storage-retrieval model this supposition is examined for its empirical soundness. First to be discussed is whether with interpolation the presentation mode influences storage and retrieval in memory. The question of whether a distributed mode can induce storage loss is emphasized here. Second, it is to be discussed whether Batchelder and Riefer's (1980) two-factor hypothesis can be generalized to retroactive inhibition. This hypothesis postulates a smaller probability for storage and a larger probability for retrieval when categorizable word pairs are presented in a distributed manner.

A free recall experiment is reported with two experimental factors: interpolation level (1-5 lists presented) and presentation mode (massed versus distributed). For the massed mode the data analysis shows a retrieval failure without storage loss, while for the distributed mode some storage loss occurred. These data reveal that presentation mode does indeed affect storage processes in retroactive inhibition. Furthermore, when interpolated lists are presented, distributed presentation reduces storage probability, while retrieval probability remains unaffected. Batchelder and Riefer's two-factor hypothesis, therefore, cannot be generalized to retroactive inhibition.
\end{abstract}

\section{Introduction}

The inhibition in recall of material when other material presented at a later time is interpolated, is called retroactive inhibition. Modern

* I am grateful to J.G.W. Raaijmakers and W.H. Batchelder for their comments on an earlier draft of this paper.

Author's address: K.-H. Bäuml, Department of Psychology, Regensburg University, 8400 Regensburg, Germany. E-mail: baeuml@vax1.rz.uni-regensburg.dbe.de 
theories of retroactive inhibition assume that the inhibition is essentially retrieval-based; that is, interfered items remain stored in memory. Indeed, a lot of data support retrieval-based theories of retroactive inhibition (Tulving and Psotka 1971; Riefer and Batchelder 1988). However, in some studies there is evidence that interpolation can also cause storage loss (Cofer et al. 1971; Earhard 1976; Reynolds 1977). Their data suggest that retrieval-based theories are at best a simplification.

In a number of studies the experimental method has involved the presentation of categorizable items in order to provide evidence on the role of storage and retrieval processes in this kind of interference paradigm (Tulving and Psotka 1971; Riefer and Batchelder 1988). The items that belong to one category are usually presented massed, that is, with no intervening items between them. However, some studies demonstrate that, when no interpolated material is employed, the presentation mode of categorizable items can affect recall (Cofer et al. 1966; Borges and Mandler 1972; Glanzer 1969). Batchelder and Riefer (1980) examine whether the presentation mode of categorizable items affects storage and retrieval processes, and whether it affects them differentially. They postulate a two-factor hypothesis: (i) storage of categorizable items is more likely when the items belonging to one category are presented massed; (ii) retrieval of categorizable items that are stored together is more likely when the items belonging to one category are presented in a distributed manner. Their data strongly support this hypothesis. There are several theoretical explanations for this empirical finding. For example, the better storage for the massed mode may be explained by a more effective organization of the categorizable items (D'Agostino 1969), by an easier determination of the relevant principles of organization (Kintsch 1970), or by an easier concept identification by means of forming word associations (Glanzer 1969). The storage effect may also be explained by a two-store memory model (Atkinson and Shiffrin 1968): in the massed mode the presentation of categorizable items induces their simultaneous presence in short-term store (rehearsal buffer), while in the distributed mode the same need not be true. To make things easier, suppose that categories are of extension two. In the distributed mode, to store a category's items as a cluster the presentation of the category's second item may have to serve as a cue to retrieve the category's first item from long-term store into short-term store. This retrieval, however, can fail preventing storage of 
these items as a cluster. This reduces the probability that a category's items are stored as a cluster in the distributed mode. The retrieval effect may be explained by the hypothesis that a distributed presentation of categorizable items enhances the number of nonredundant retrieval cues (Melton 1970). This again may be caused by the usually smaller context overlap between categorizable items in the distributed mode (Raaijmakers and Shiffrin 1981).

The effect of presentation mode on retroactive inhibition has been largely neglected. There seems to be neither an experiment in the literature nor any theory of retroactive inhibition that makes explicit statements about an influence of this factor. As a consequence, no clear predictions can be deduced about an influence of presentation mode. This current state reflects the supposition that presentation mode does not play any substantial role in retroactive inhibition. This supposition appears premature. On the one hand, the empirical results reviewed provide no evidence on this problem; in studies on retroactive inhibition categorizable words are usually presented massed. On the other hand, the empirical results reviewed suggest an important role of presentation mode in storage and retrieval processes. A priori it is therefore not at all clear whether the differences in storage and retrieval that are due to presentation mode remain constant when interpolated material is presented. In this article it is examined whether the neglect of presentation mode in retroactive inhibition is empirically justified.

A free recall experiment is reported with two independent experimental factors: interpolation level (1-5 lists presented) and presentation mode (massed versus distributed). The lists contain conceptually categorizable word pairs. By applying Batchelder and Riefer's (1980) storage-retrieval model, it is tested how these two factors influence storage and retrieval processes. First, for both presentation modes, it is tested whether interpolation affects storage and/or retrieval. Here the question is emphasized whether the distributed presentation of the categorizable items can induce storage loss. Second, for each level of interpolation, it is tested whether the storage and retrieval processes behave similarly across presentation modes. This test addresses the question of whether Batchelder and Riefer's (1980) two-factor hypothesis may be generalized to retroactive inhibition. 


\section{Method}

\section{Batchelder and Riefer's storage-retrieval model}

The storage-retrieval model proposed by Batchelder and Riefer $(1980,1986)$ enables the separate measurement of a storage and a retrieval parameter. It is especially appropriate for an application to retroactive inhibition. For each level of interpolation the storage and the retrieval parameter are measured. Storage loss is diagnosed if the storage parameter declines with the number of interpolated lists, retrieval failure is diagnosed if the retrieval parameter declines with the number of interpolated lists. The model is formulated for the analysis of data from free recall experiments. The material consists of both categorizable item pairs and noncategorizable single items. The data of an experiment can be described by the following joint sample space:

$E_{1}$ - both items of a categorizable pair are recalled, adjacently;

$E_{2}$ - both items of a categorizable pair are recalled, nonadjacently;

$E_{3}$ - one and only one item of a categorizable pair is recalled;

$E_{4}$ - none of the items of a categorizable pair is recalled;

$F_{1}$ - a single item is recalled;

$F_{2}$ - a single item is not recalled.

The pair observations are assumed to be independent and identically distributed over $E_{i}(i=1,2,3,4)$. So they can be described by a multinomial distribution with the parameters $p\left(E_{i}\right)$, where $p\left(E_{i}\right)$ is interpreted as the probability of the occurrence of the event $E_{i}$. By the same principle, the observations falling into $F_{j}(j=1,2)$ are assumed to be independent and identically distributed. They can therefore be described by a binomial distribution with the parameters $q\left(F_{j}\right)$, where $q\left(F_{j}\right)$ is interpreted as the probability of the occurrence of the event $F_{j}$. The likelihood function of the data observations under this statistical multinomial model is:

$L_{S}=\frac{N !}{N_{1} ! N_{2} ! N_{3} ! N_{4} !} p\left(E_{1}\right)^{N_{1}} p\left(E_{2}\right)^{N_{2}} p\left(E_{3}\right)^{N_{3}} p\left(E_{4}\right)^{N_{4}} \frac{M !}{M_{1} ! M_{2} !} q\left(F_{1}\right)^{M_{1}} q\left(F_{2}\right)^{M_{2}}$

Batchelder and Riefer (1986) postulate three cognitive processes: a storage process for pairs, a retrieval process for stored pairs and a storage-and-retrieval process for singles and pairs not stored as clusters. The following six assumptions are made (cf. Batchelder and Riefer 1986):

A1. (Storage of clusters) A pair either is or is not stored as a cluster. Let $c$ be the probability that a pair is stored as a cluster $(0 \leq c \leq 1)$.

A2. (Retrieval of clusters) If a pair is stored as a cluster, it either is or is not retrieved as a cluster. Let $r$ be the conditional probability that a pair is recalled as a cluster, given that it is stored as a cluster $(0 \leq r \leq 1)$. 
A3. (Retrieval of nonclustered pairs) If a pair is not stored as a cluster, each item either is or is not stored and retrieved independently. Let $u$ be the probability that a nonclustered item is recalled $(0 \leq u \leq 1)$.

A4. (Recall of pairs) The retrieval of a cluster results in the adjacent recall of both category members $\left(E_{1}\right)$, while the nonretrieval of a cluster leads to the nonrecall of both items $\left(E_{4}\right)$. The recall of a nonclustered pair results either in the recall of both items nonadjacently $\left(E_{2}\right)$, or in the recall of one and only one item $\left(E_{3}\right)$, or in the recall of none of both items $\left(E_{4}\right)$.

A5. (Storage and retrieval of singles) Each single item either is or is not stored and retrieved; again, let $u$ be the probability for this event.

A6. (Recall of singles) If a single item is stored and retrieved, it is recalled $\left(\mathrm{F}_{1}\right)$, if not. it is not recalled $\left(F_{2}\right)$.

These assumptions bear some strong implications. For example, it is implied that the parameters $c, r, u$ are constant for all pairs and singles, respectively; it is implied that there are no interactions between pairs, singles or both; it is further implied that the parameters for the storage-and-retrieval of singles and the storage-and-retrieval of items of nonclustered pairs are identical $(u)$; finally, it is implied that the three cognitive processes are all all-or-none-processes: there are no intermediate states. Note that A4 contains a simplification: it implicitly assumes that the adjacent recall of a pair can only be caused by a successful retrieval of a stored cluster, that is, it neglects the possibility of a chance adjacent recall. Note also that parameter $r$ represents the combined result of two processes: accessing the cluster and recall of the items given access to the cluster. The model equations relating the data observations and the cognitive processes can be easily derived from the assumption of the model:

$$
\begin{aligned}
& p\left(E_{1}\right)=c r ; \\
& p\left(E_{2}\right)=(1-c) u^{2} ; \\
& p\left(E_{3}\right)=(1-c) 2 u(1-u) ; \\
& p\left(E_{4}\right)=c(1-r)+(1-c)(1-u)^{2} ; \\
& q\left(F_{1}\right)=u ; \\
& q\left(F_{2}\right)=1-u .
\end{aligned}
$$

From the resulting likelihood function of the model

$$
\begin{aligned}
L_{C}= & \frac{N !}{N_{1} ! N_{2} ! N_{3} ! N_{4} !}[c r]^{N_{1}}\left[(1-c) u^{2}\right]^{N_{2}}[(1-c) 2 u(1-u)]^{N_{3}} \\
& \times\left[c(1-r)+(1-c)(1-u)^{2}\right]^{N_{4}} \frac{M !}{M_{1} ! M_{2} !} u^{M_{1}}(1-u)^{M_{2}},
\end{aligned}
$$


(where $N_{i}$ is the absolute frequency of the event $E_{i}, i=1,2,3,4, M_{j}$ that of $F_{j}, j=1$, 2. with $N=\sum N_{i}, M=\sum M_{j}$ ) explicit maximum-likelihood estimates of the parameters can be deduced (cf. Batchelder and Riefer 1986: 132).

Note that the storage-retrieval model is not a model of retroactive inhibition. Instead it is a model to describe data from single experimental conditions. If the application of the model is successful it may serve as a measurement tool to examine hypotheses about storage and retrieval processes like in retroactive inhibition (see Statistical analysis for details).

\section{Subjects}

One hundred psychology students at the University of Regensburg participated in the experiment. The subjects were randomly assigned to the experimental conditions. They were not paid for their participation, but received credit for fulfilling a degree requirement. None of the subjects had previous experience with free recall experiments.

\section{Material and apparatus}

The word material consisted of 125 items. 100 of these items were different conceptually categorizable pairs (for example, translated into English: apple-pear, rain-snow, cello-trombone, etc.). The remaining 25 items were so-called singles, that is, items that are not categorizable in an obvious manner, neither mutually nor with regard to the pairs. The pairs and the singles were largely taken from the categories of Battig and Montague (1969), although some categories not found in Battig and Montague were also included in the lists. On the other hand, some of their categories were ignored, because they are unusual to Germans. For every category we tried to take the two (pairs) or the one (singles) most prominent category word(s). The pairs were therefore highly associated. Furthermore, the words were selected to minimize the possibility of categorization in an alternative way, for example phonetically-visually (rhymes). The 125 items were randomly assigned to five lists under the restriction that in every list there were 10 pairs and 5 singles. So every list consisted of 25 items. There was a new random assignment of the items to the five lists after half of the subjects had participated in the experiment.

\section{Design and procedure}

There were two controlled experimental factors, interpolation level and presentation mode. The interpolation level varied from one presented list (no interpolation) to five presented lists. The presentation mode was either massed or distributed. The subjects were randomly assigned to the ten experimental conditions. Subjects were tested individually. At the beginning of each experimental session the items within a list were mixed randomly. For the massed mode this was done under the restriction that the pairs are presented with no intervening items, for the distributed mode there was no such restriction. The items were presented on the screen one at a time. They were presented at an exposure rate of $5 \mathrm{sec}$ per item. After the presentation of a complete list 
subjects were given $90 \mathrm{sec}$ to write down the 25 items from this list. After this recall period and a break of $60 \mathrm{sec}$, the session continued with the next list. After all of the lists had been presented, a final free recall test was given in which subjects attempted to recall the items from all of the previous lists. Subjects were given up to 5 minutes for this final written recall. The time intervals turned out to be sufficient for recall. Nearly all subjects were finished before time elapsed. The subjects were instructed to guess when they were not sure whether an item had been presented. They were to recall as many items as possible. This procedure is very similar to the experimental conditions employed by Tulving and Psotka (1971) and Riefer and Batchelder (1988).

\section{Statistical analysis}

For all ten experimental conditions, the first list final recall was analyzed by means of Batchelder and Riefer's (1980) storage-retrieval model. First, it was tested whether the model fits the data sets from all the experimental conditions. This was done by means of a likelihood-ratio test. This test examines whether the model fits the experimental data equally well as the statistical multinomial model. The test is based on the well-known fact that, if the model is true, $-2 \ln L_{C} / L_{S}$ is asymptotically distributed as a $\chi^{2}$-random variable with degrees of freedom given by the number of parameters free to vary in the statistical parameter space minus the number of parameters free to vary in the model's parameter space (cf. Lindgren 1976). An $\alpha=0.05$ was selected.

If the model fitted the single data sets, for both presentation modes it was tested whether the parameters $c, r$ and $u$ could be assumed to be constant across interpolation levels. Again this was done by performing a likelihood-ratio test. Across all five interpolation levels it was tested whether a more restrictive model ( $c=$ const or $r=$ const or $u=$ const with 11 parameters respectively) fits the data as well as the unrestricted model (15 parameters). The test is quite analogous to the likelihood-ratio test examining the storage-retrieval model. Furthermore, for all five interpolation levels, it was tested whether the parameters $c, r$ and $u$ are identical across presentation modes. First, the constancy of parameter $c$ was tested for variable parameters $r$ and $u$ (five parameters), then the constancy of parameter $r$ was tested for variable parameters $c$ and $u$ (five parameters), and, finally, the constancy of parameter $u$ was tested for variable parameters $c$ and $r$ (five parameters). In each case a likelihood-ratio test was conducted comparing the more restrictive models against the unrestricted model (six parameters). In all of these cases the parameters were estimated by numerical optimizations. Here the likelihood functions were optimized under the restriction that the parameters lie within the real interval $[0,1]$.

\section{Results}

Tables 1 and 2 show the estimated probabilities (relative frequencies) - that is, the parameter estimations for the statistical multinomial model - for each observation category in the two presentation modes. As the number of interpolated lists is 
Table 1

Massed mode: Estimated probabilities (relative frequencies) for the observation categories $E_{i}$ $(i=1,2,3,4)$ and $F_{j}(j=1,2)$ for all five interpolation levels.

\begin{tabular}{lllllll}
\hline $\begin{array}{l}\text { Number } \\
\text { of lists }\end{array}$ & $p\left(E_{1}\right)$ & $p\left(E_{2}\right)$ & $p\left(E_{3}\right)$ & $p\left(E_{4}\right)$ & $q\left(F_{1}\right)$ & $q\left(F_{2}\right)$ \\
\hline 1 & 0.69 & 0.02 & 0.05 & 0.24 & 0.62 & 0.38 \\
2 & 0.58 & 0.04 & 0.06 & 0.32 & 0.50 & 0.50 \\
3 & 0.55 & 0.01 & 0.03 & 0.41 & 0.60 & 0.40 \\
4 & 0.48 & 0.02 & 0.03 & 0.47 & 0.48 & 0.52 \\
5 & 0.39 & 0.03 & 0.06 & 0.52 & 0.44 & 0.56 \\
\hline
\end{tabular}

increased, for both modes of presentation the data show a decline in the probability that a pair is recalled with no intervening items $\left(p\left(E_{1}\right)\right)$ and an increase in the probability that a pair is not recalled at all $\left(p\left(E_{4}\right)\right)$. Also for both modes the data show a decline in the probability that a single is recalled $\left(q\left(F_{1}\right)\right)$ with increasing interpolation level. Moreover, there is hardly a difference between modes of presentation in the probability that the members of a pair are recalled nonadjacently $\left(p\left(E_{2}\right)\right)$; for both modes this probability is very small and fairly constant across interpolation levels. However, the data also differ in some respects. Both the decline in the probability that a pair is recalled adjacently $\left(p\left(E_{1}\right)\right)$ and the increase in the probability that a pair is not recalled at all $\left(p\left(E_{4}\right)\right)$ are smaller for the massed mode than for the distributed mode. Finally, while the probability for the recall of just one word of a pair $\left(p\left(E_{3}\right)\right)$ remains fairly constant for the massed mode, it increases somewhat for the distributed mode. Thus these statistics suggest differences both between interpolation levels and between presentation modes.

Table 3 shows the parameter estimations for $c, r$ and $u$ and the $\chi^{2}$-values from the likelihood-ratio tests, comparing the storage-retrieval model with the statistical multinomial model for the massed mode. The storage-retrieval model has three parameters and describes four independent data observations. So the test is conducted with one degree of freedom. As can be seen, the fit of the model is excellent, with none of the data sets leading to a rejection of the model.

Table 2

Distributed mode: Estimated probabilities (relative frequencies) for the observation categories $E_{i}$ $(i=1,2,3,4)$ and $F_{j}(j=1,2)$ for all five interpolation levels.

\begin{tabular}{lcccccc}
\hline $\begin{array}{l}\text { Number } \\
\text { of lists }\end{array}$ & $p\left(E_{1}\right)$ & $p\left(E_{2}\right)$ & $p\left(E_{3}\right)$ & $p\left(E_{4}\right)$ & $q\left(F_{1}\right)$ & $q\left(F_{2}\right)$ \\
\hline 1 & 0.73 & 0.07 & 0.09 & 0.11 & 0.48 & 0.52 \\
2 & 0.62 & 0.07 & 0.04 & 0.27 & 0.46 & 0.54 \\
3 & 0.48 & 0.04 & 0.07 & 0.41 & 0.38 & 0.62 \\
4 & 0.28 & 0.08 & 0.14 & 0.50 & 0.34 & 0.66 \\
5 & 0.30 & 0.04 & 0.15 & 0.51 & 0.28 & 0.72 \\
\hline
\end{tabular}


Table 3

Massed mode: Parameter estimations for storage parameter $c$, retrieval parameter $r$ and parameter $u$ and $\chi^{2}$-values from the likelihood-ratio tests comparing the storage-retrieval model with the statistical multinomial model for all five interpolation levels.

\begin{tabular}{lllll}
\hline $\begin{array}{l}\text { Number } \\
\text { of lists }\end{array}$ & \multicolumn{2}{l}{ Parameter } & & $\chi^{2}(1)$ \\
\cline { 2 - 4 } & $c$ & $r$ & $u$ & \\
\hline 1 & 0.917 & 0.753 & 0.600 & 0.693 \\
2 & 0.869 & 0.668 & 0.511 & 0.164 \\
3 & 0.952 & 0.578 & 0.587 & 0.514 \\
4 & 0.932 & 0.515 & 0.488 & 0.144 \\
5 & 0.871 & 0.448 & 0.448 & 0.098 \\
\hline
\end{tabular}

Fig. 1 shows the parameter estimates for $c$ and $r$ graphically as a function of the number of presented lists. Despite some oscillations $c$ appears to be relatively constant, while parameter $r$ declines strongly. The likelihood-ratio tests examining the constancy of the parameters are conducted with four degrees of freedom each. The tests show that the parameter $c$ does not significantly differ across interpolation levels reaching a value of $\chi^{2}(4)=4.848$; so $c$ can be assumed to be constant. On the other hand, the tests

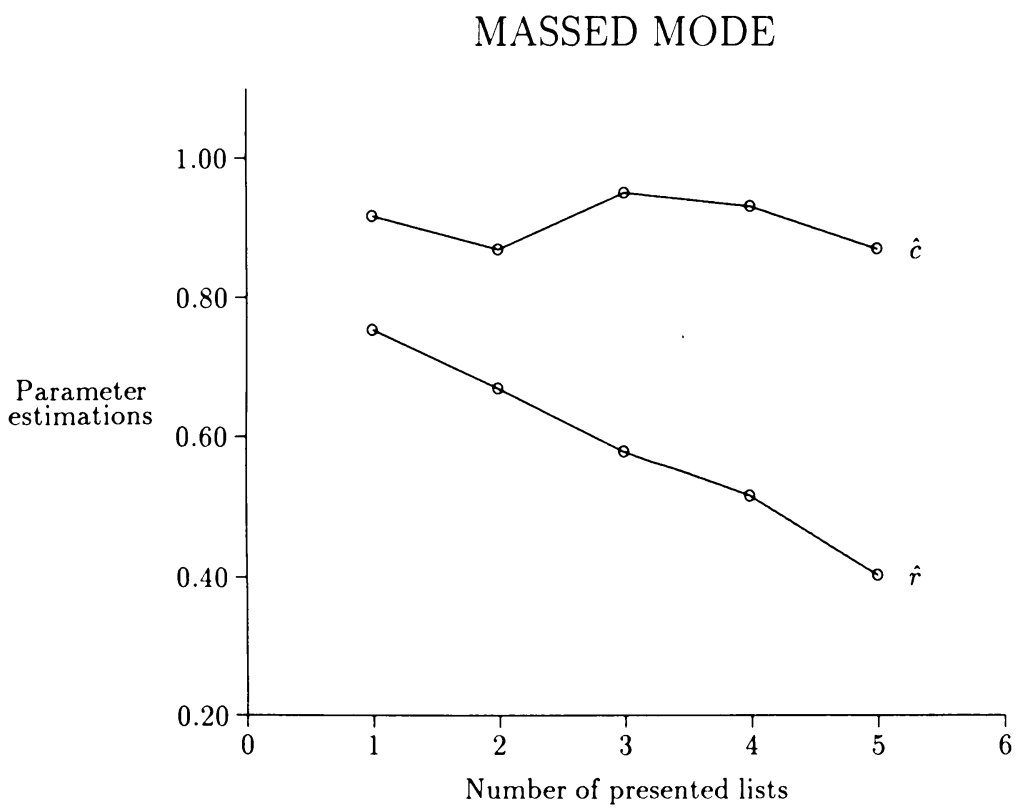

Fig. 1. Massed mode: Graphical representation of the storage parameter $c$ and the retrieval parameter $r$ as a function of the number of presented lists. 
Table 4

Distributed mode: Parameter estimations for storage parameter $c$, retrieval parameter $r$ and parameter $u$ and $\chi^{2}$-values from the likelihood-ratio tests comparing the storage-retrieval model with the statistical multinomial model for all five interpolation levels.

\begin{tabular}{lllll}
\hline $\begin{array}{llll}\text { Number } \\
\text { of lists }\end{array}$ & \multicolumn{2}{l}{ Parameter } & & $\chi^{2}(1)$ \\
\cline { 2 - 4 } & $c$ & $r$ & $u$ & \\
\hline 1 & 0.790 & 0.925 & 0.510 & 0.801 \\
2 & 0.857 & 0.723 & 0.522 & $4.405^{\mathrm{a}}$ \\
3 & 0.829 & 0.579 & 0.404 & 0.777 \\
4 & 0.651 & 0.430 & 0.393 & 2.144 \\
5 & 0.622 & 0.483 & 0.294 & 0.232 \\
\hline
\end{tabular}

${ }^{\mathrm{a}} \alpha<0.05$.

show that the decline of parameter $r$ is a statistically reliable effect reaching a value of $\chi^{2}(4)=20.664$; so $r$ cannot be assumed to be constant. Parameter $u$ appears to oscillate and to decrease slightly. However, the decline does not pass the level of significance $\left(\chi^{2}(4)=7.068\right)$, so the parameter can be assumed to be constant.

\section{DISTRIBUTED MODE}

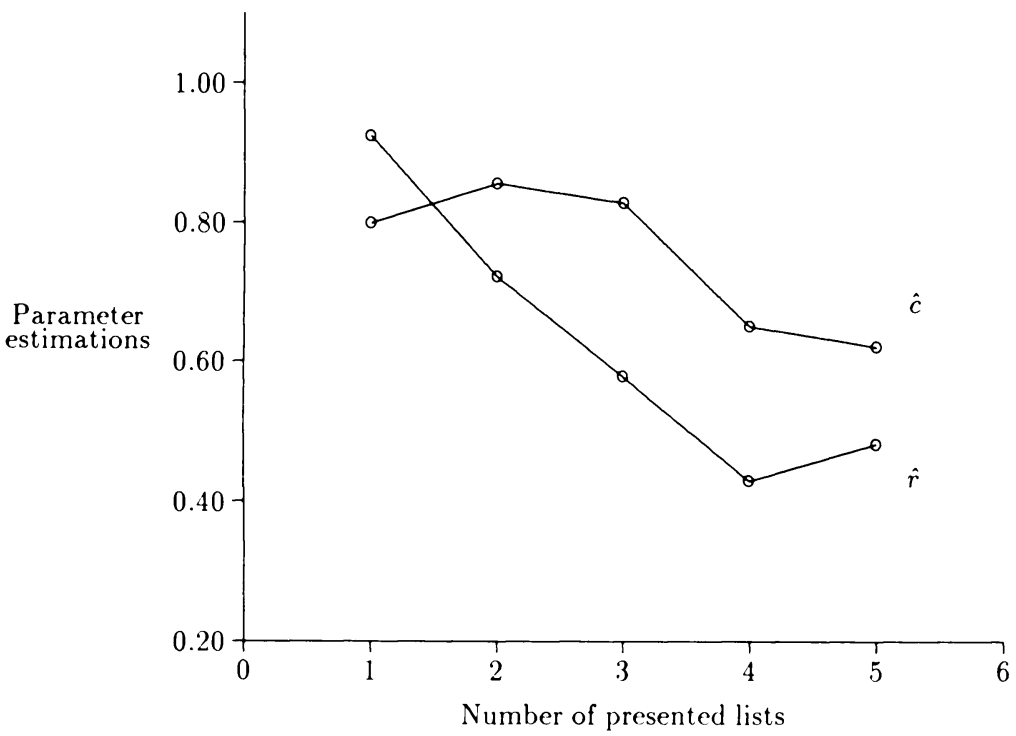

Fig. 2. Distributed mode: Graphical representation of the storage parameter $c$ and the retrieval parameter $r$ as a function of the number of presented lists. 
Table 5

$\chi^{2}$-values from the statistical tests examining the identity of storage parameter $c$, retrieval parameter $r$ and parameter $u$ across presentation modes, for the five interpolation levels.

\begin{tabular}{llll}
\hline $\begin{array}{l}\text { Number } \\
\text { of lists }\end{array}$ & $\chi^{2}$-values & \\
\cline { 2 - 4 } & $c=$ const & $r=$ const & $u=$ const \\
\hline 1 & $4.844^{\mathrm{a}}$ & $6.901^{\mathrm{b}}$ & 1.005 \\
2 & 0.036 & 0.546 & 0.031 \\
3 & $5.439^{\mathrm{a}}$ & 0.001 & 3.804 \\
4 & $14.174^{\mathrm{b}}$ & 0.823 & 1.133 \\
5 & $6.937^{\mathrm{b}}$ & 0.110 & 3.075 \\
\hline
\end{tabular}

${ }^{\mathrm{a}} \alpha<0.05 ;{ }^{\mathrm{b}} \alpha<0.01$.

Table 4 shows the parameter estimates for $c, r$ and $u$ and the $\chi^{2}$-values from the likelihood-ratio tests, comparing the storage-retrieval model with the statistical model for the distributed mode. Again the tests are conducted with one degree of freedom. As can be seen the fit of the model is very good with the exception of interpolation level two, which just passes the level of significance. However, because the deviation for level two is not very strong, I will include this condition in the further analyses.

A graphical representation of the parameter estimates for $c$ and $r$ (fig. 2) suggests that the results for both presentation modes differ in some respects. This is mainly due to parameter $c$. While remaining fairly constant for the first three interpolation levels, $c$ declines abruptly for the last two interpolation levels. Again parameter $r$ declines strongly with the number of presented lists, however, showing a slight increase for the last list condition. The respective likelihood-ratio tests examining the reliability of these oscillations show that both the decrease of $c$ and the decrease of $r$ are indeed statistically reliable effects $\left(\chi^{2}(4)=10.894\right.$ and $\chi^{2}(4)=29.335$, respectively). With four degrees of freedom none of the parameters can be assumed to be constant across interpolation levels. Parameter $u$ also decreases across interpolation levels. It also cannot be regarded as constant $\left(\chi^{2}(4)=12.335\right)$.

To test whether Batchelder and Riefer's two-factor hypothesis can be generalized to retroactive inhibition the two presentation modes are compared. At each interpolation level it is tested whether the parameters $c$ and $r$ can be assumed to be identical across presentation modes. Table 5 shows the $\chi^{2}$-values resulting from the respective likelihood-ratio tests. For four of the five interpolation levels constancy of parameter $c$ has to be rejected. Only for the two-list condition the constancy of the parameter can be assumed; however, note that for this condition the fit of the model is not as good as for the other conditions (compare above). The analysis of parameter $r$ leads to completely different results. For four of the five interpolation levels the constancy of the parameter need not be rejected. It is only the one-list condition that cannot be described using the same $r$ for both presentation modes. So the theoretical description of the one-list condition requires different sets of $c$ 's and $r$ 's to account for the two presentation modes, while for the two-list condition the same set would do. The conditions in which three, four and five lists have been presented can be described with an identical $r$, 
however, all three need different variables $c$ to handle the two presentation modes. So the difference between massed and distributed presentation of word pairs is mainly reflected in the storage parameter $c$ and hardly in the retrieval parameter $r$.

\section{Discussion}

For the massed mode the data analysis shows that all five interpolation levels can be described excellently by the storage-retrieval model. This result is in accordance with that of Riefer and Batchelder (1988). Thus the model's parameter estimates can be used to examine the effect of interpolation on storage and retrieval processes. Retrieval-based theories of retroactive inhibition assume no storage loss due to interpolation. In the context of the storage-retrieval model this assumption is identical with the restriction of the storage parameter to be independent of interpolation level. Indeed the respective likelihood-ratio tests support this assumption: the storage parameter shows no reliable variations with interpolation level. The same is not true for the retrieval parameter that declines with interpolation levels. This result suggests a retrieval failure. So, with categorizable items being presented in a massed fashion, interpolation leads to a retrieval failure while there is no storage loss. This result agrees with Riefer and Batchelder's (1988) data and with the conclusions drawn by Tulving and Psotka (1971). It is in accordance with retrieval-based theories of retroactive inhibition.

For the distributed mode the data sets are also quite well described by the storage-retrieval model. Only for the two-list condition do the data slightly deviate from the model's predictions. In contrast to the massed mode, in the distributed presentation mode neither the storage parameter nor the retrieval parameter can be assumed to be constant across interpolation levels. The strong decline of the retrieval parameter suggests a retrieval failure as the number of presented lists is increased. While the storage parameter is relatively constant for the first three interpolation levels, there is an abrupt decrease with the fourth interpolation level. This pattern suggests a storage loss. Thus in case of a distributed presentation retroactive inhibition results in both a retrieval failure and a storage loss. This result is in conflict with retrieval-based theories.

In an experiment that did not employ interpolated material, Batchelder and Riefer (1980) found presentation mode to affect storage 
and retrieval processes in the way summarized in their two-factor hypothesis. The present data confirm this hypothesis: in the respective likelihood-ratio tests the storage parameter is larger and the retrieval parameter is smaller in the massed versus the distributed presentation mode. With respect to storage this result can be generalized to interpolation: the likelihood-ratio tests show that for all five interpolation levels there is a greater storage parameter for the massed than for the distributed mode. The same does not hold with respect to retrieval. The tests find no reliable differences in the retrieval parameters when the two presentation modes are compared at the respective interpolation levels. This result suggests that, with interpolation, there is no difference in retrieval between presentation modes. Thus the better retrievability for the distributed mode found for the non-interpolation condition cannot be generalized to interpolation. Batchelder and Riefer's (1980) two-factor hypothesis therefore cannot be generalized to retroactive inhibition. Instead, under conditions of retroactive inhibition it reduces to a one-factor hypothesis describing the effect of presentation mode on storage processes. The strength of this effect again is a function of interpolation level.

The present results demonstrate that the presentation mode affects storage processes in retroactive inhibition: in the massed mode there is no storage loss, the distributed mode induces some storage loss. This is a rather surprising result. It reveals that the supposition that presentation mode does not affect retroactive inhibition does not hold empirically.

The question arises, what is actually happening with clusters that are 'lost' from the memory store. By applying the storage-retrieval model the present data suggest an answer to this question. For the distributed mode the results show that, for interpolation levels four and five, some clusters are lost, that is, some pairs that have been clustered in lower interpolation levels are no longer stored as a cluster in memory. In the storage-retrieval model the items from these lost clusters are handled in just the same way as singles and items from pairs that are not clustered: they are all handled by parameter $u$. If this restriction is not true empirically, the storage-retrieval model will not fit the data demanding different parameters for these kinds of items. However, this is not what has been found in the likelihood-ratio tests; the tests do not give rise to a separate theoretical handling of these items. As a consequence, there is no reason to assume that items from lost clusters 
behave differently than singles or items from pairs that are not clustered. This suggests, at least as a first approximation, that the fate of a lost cluster simply is to decay into its single items.

The next question that arises is how the distributed mode might induce such a decay. The better storage in the massed mode without interpolation has been explained by an easier concept identification, an easier determination of the relevant principles of organization, or a more effective organization of the categorizable items. By means of a two-store memory model the same effect has been explained by a possible retrieval failure between categorizable items in distributed mode. The storage loss in the distributed mode may also be explained by means of a two-store memory model. In the distributed mode the retrieval of a category's first item from long-term store when the category's second item is presented usually will not be immediate. If we take the amount of information stored about these two items to be proportional to the time that the two items are simultaneously present in the rehearsal buffer (Raaijmakers and Shiffrin 1981), there should be a smaller interitem associative strength in the distributed mode than in the massed mode. In addition, the smaller context overlap of the category's items in the distributed mode may further enhance the difference in interitem associative strength between the two modes. Suppose that for every cluster there is some probability of decay into its singles, and suppose that this probability is a negative function of the interitem associative strength. Then we would expect a greater probability for a cluster's decay in the distributed mode than in the massed mode. This pattern is suggested in the data.

Without interpolation, presentation mode affects retrieval processes. This pattern does not hold in retroactive inhibition: with interpolation no differences in retrieval can be found across presentation modes. The more complete retrieval in the distributed mode without interpolation may be explained by an enhancement of the number of nonredundant cues. Because the nonredundant cues are also exposed to a retrieval failure with interpolation the above explanation leads to the expectation that the more complete retrieval for the distributed mode should gradually become smaller and smaller with interpolation. This pattern is at least roughly present in the data, although the decline may be somewhat faster than expected. However, note that parameter $r$ reflects a conditional probability depending on parameter $c$, and that its estimates therefore are not as good as for parameter $c$. The power to 
detect differences about $r$ is also lower than the power to detect differences about $c$ (Riefer and Batchelder 1990).

For the present examination parameter $u$ of the storage-retrieval model is not of strong interest because it confounds storage and retrieval processes for singles and not clustered items, while here we are mainly interested in the effect of presentation mode on storage and retrieval processes. In addition, the presentation of singles and items that are not clustered is not all contingent upon presentation mode. One would therefore expect this parameter to be constant across presentation modes. At first sight this expectation seems to be violated: while the likelihood-ratio tests have found $u$ to be constant for the massed mode, for the distributed mode they have found $u$ to decline significantly. However, a direct comparison of the single interpolation levels across presentation modes reveals that the difference across modes is not significant (table 5): for none of the five interpolation levels the assumption of a constant $u$ has to be rejected. Although for levels three and five the difference is almost significant, the pattern as a whole does not support the assumption of a systematic difference. Thus, as expected, parameter $u$ can be assumed to be constant across presentation modes.

The storage-retrieval model does an excellent job in fitting the single data sets. Although it is not a model of retroactive inhibition, it is a very useful tool for theorizing about storage and retrieval processes. One of its main advantages is that it enables a separate analysis of storage and retrieval processes. Storage loss and retrieval failure can be defined in a natural way. Using conventional statistics the same cannot be achieved with the same elegancy. The use of conventional statistics

Table 6

Conventional statistics for massed mode and distributed mode: $P(c)$ - probability for an item to be recalled, $P($ cat $)$ - probability for a category to be recalled, IPC - probability for an item to be recalled when its category is recalled.

\begin{tabular}{llllllll}
\hline $\begin{array}{l}\text { Number } \\
\text { of lists }\end{array}$ & \multicolumn{2}{l}{ Massed mode } & & \multicolumn{3}{l}{ Distributed mode } \\
\cline { 2 - 3 } & $P(c)$ & $P($ cat $)$ & $I P C$ & & $P(c)$ & $P($ cat $)$ & $I P C$ \\
\hline 1 & 0.735 & 0.760 & 0.967 & & 0.845 & 0.890 & 0.949 \\
2 & 0.650 & 0.680 & 0.956 & & 0.710 & 0.730 & 0.973 \\
3 & 0.575 & 0.590 & 0.975 & & 0.555 & 0.590 & 0.941 \\
4 & 0.515 & 0.530 & 0.972 & & 0.430 & 0.500 & 0.860 \\
5 & 0.450 & 0.480 & 0.938 & & 0.415 & 0.490 & 0.847 \\
\hline
\end{tabular}


is also less well founded and can, as Riefer and Batchelder (1988) show, lead to wrong implications about storage and retrieval processes (I have conducted some not yet published experiments where this is indeed the case). Nevertheless, with the present data the usage of conventional statistics would suggest roughly the same conclusions as have been derived by means of the storage-retrieval model (table 6). So the decline in the probability of a category to be recalled $(P($ cat $))$ agrees very well with the decline in the retrieval parameter $r$, while the constant (declining) probability of an item to be recalled when its category is recalled (IPC) for the massed (distributed) mode agrees at least roughly with the respective constancy (decline) of the storage parameter $c$. This agreement between the storage-retrieval model and the conventional statistics, however, was not guaranteed a priori. Finally, note that the pattern of results with respect to the storage processes is much clearer with the storage-retrieval model than by using conventional statistics.

\section{References}

Atkinson, R.C. and R.M. Shiffrin, 1968. 'Human memory: A proposed system and its control processes'. In: K.W. Spence and J.T. Spence (eds.), The psychology of learning and motivation: Advances in research and theory, Vol. 2. New York: Academic Press.

Batchelder, W.H. and D.M. Riefer, 1980. Separation of storage and retrieval factors in free recall of clusterable pairs. Psychological Review 87, 375-397.

Batchelder, W.H. and D.M. Riefer, 1986. The statistical analysis of a model for storage and retrieval processes in human memory. British Journal of Mathematical and Statistical Psychology 39, 129-149.

Battig, W.F. and W.E. Montague, 1969. Category normes for verbal items in 56 categories: A replication and extension of the Connecticut category norms. Journal of Experimental Psychology 80 (3, Pt. 2), 1-46.

Borges, M.A. and G. Mandler, 1972. Effect of within-category spacing on free recall. Journal of Experimental Psychology 92, 207-214.

Cofer, C.N., D.R. Bruce and G.M. Reicher, 1966. Clustering in free recall as a function of certain methodological variations. Journal of Experimental Psychology 71, 858-866.

Cofer, C.N., N.F. Failie and D.L. Horton, 1971. Retroactive inhibition following reinstatement or maintenance of first-list responses by means of free recall. Journal of Experimental Psychology 90, 197-205.

D'Agostino, P.R., 1969. The blocked-random effect in recall and recognition. Journal of Verbal Learning and Verbal Behavior 8, 815-820.

Earhard, B., 1976. Retroactive inhibition: Associative responses or response suppression? American Journal of Psychology 89, 385-401.

Glanzer, M., 1969. Distance between related words in free recall: Traces of the STS. Journal of Verbal Learning and Verbal Behavior 8, 105-111. 
Kintsch. W., 1970. Learning, memory, and conceptual processes. New York: Wiley.

Lindgren, B.W., 1976. Statistical theory (3rd ed.). New York: Macmillan.

Melton, A.W., 1970. The situation with respect to the spacing of repetitions and memory. Journal of Verbal Learning and Verbal Behavior 9, 1-21.

Raaijmakers, J.G.W. and R.M. Shiffrin, 1981. Search of associative memory. Psychological Review 88, 93-134.

Reynolds, J.H., 1977. Unavailable and inaccessible information in retroactive inhibition of paired associates. Journal of Experimental Psychology: Human Learning and Memory 3, 68-77.

Riefer, D.M. and W.H. Batchelder, 1988. Multinomial modeling and the measurement of cognitive processes. Psychological Review 95, 318-339.

Riefer, D.M. and W.H. Batchelder, 1990. Statistical inference for multinomial processing tree models. Irvine Research Unit in Mathematical Behavioral Sciences. Technical Report Series MBS 90-05.

Tulving, E. and J. Psotka, 1971. Retroactive inhibition in free recall: Inaccessibility of information available in the memory store. Journal of Experimental Psychology 87, 1-8. 